\title{
Frenkel electron and a spinning body in a curved background
}

\author{
Walberto Guzmán Ramírez, ${ }^{a}$ Alexei A. Deriglazov ${ }^{a, b}$ \\ and Andrey M. Pupasov-Maksimov ${ }^{a}$ \\ ${ }^{a}$ Depto. de Matemática, ICE, \\ Universidade Federal de Juiz de Fora, São Pedro, MG, Brasil \\ ${ }^{b}$ Laboratory of Mathematical Physics, Tomsk Polytechnic University, \\ 634050 Tomsk, Lenin Ave. 30, Russia \\ E-mail: wguzman@cbpf.br, alexei.deriglazov@ufjf.edu.br, \\ pupasov@phys.tsu.ru
}

ABSTRACT: We develop a variational formulation of a particle with spin in a curved spacetime background. The model is based on a singular Lagrangian which provides equations of motion, a fixed value of spin and Frenkel condition on spin-tensor. Comparing our equations with those of Papapetrou we conclude that the Frenkel electron in a gravitational field has the same behavior as a rotating body in the pole-dipole and leading-spin approximation. Due to constraints presented in the formulation, position space is endowed with a noncommutative structure induced by the spin of the particle. Therefore, the model provides a physically interesting example of a noncommutative particle in a curved background.

Keywords: Classical Theories of Gravity, Non-Commutative Geometry

ARXiv EPRINT: 1311.5743v1 


\section{Contents}

1 Introduction 1

2 Variational problem for Frenkel and Bargmann-Michel-Telegdi equations 3

3 Spinning particle in a curved space-time $\quad 5$

3.1 Hamiltonian constraints 6

4 Hamiltonian and Lagrangian equations of motion in curved background 9

5 First order approximation $\quad 12$

6 Conclusions 14

$\begin{array}{ll}\text { A Dirac brackets } & 14\end{array}$

\section{Introduction}

The behavior of a spin one-half elementary particle and a rotating body (spinning test particle) in an external electromagnetic or gravitational background has long been a subject of intensive study in physics. In both cases, non-relativistic theory can be described in terms of the center-of-mass and three-vector of spin $\mathbf{S}$ (for the case of a body this happens in the pole-dipole approximation $[1,2])$. Spin represents the internal angular momentum of a particle, its torque leads to precession. The first attempt to describe a spin one-half particle in relativistic theory was due to Thomas [3]; he pointed out a relativistic correction to the spin precession. Frenkel observed [4] that the natural description of spin in special relativity can be achieved by including $\mathbf{S}$ in the antisymmetric spin-tensor $J^{\mu \nu}, J^{i j}=2 \epsilon^{i j k} S^{k}$. In this case, the right balance between non-relativistic and relativistic degrees of freedom is achieved by imposing the condition $J^{\mu \nu} \dot{x}_{\nu}=0$. He also noticed that construction of a consistent system of equations (as well as of a variational problem) for position and spin-tensor, subjected to this condition, represents a rather nontrivial problem.

To describe polarization effects of an electron in uniform electromagnetic fields, Bargmann, Michael and Telegdi (BMT) [5] suggested equations of motion in terms of a fourvector of spin instead of a spin-tensor. In the linear approximation on electromagnetic field strength, the BMT equations agree with those of Frenkel.

Various variational formulations for a spin one-half particle have been proposed, ${ }^{1}$ see $[6-16]$ and references therein. In the present work, we use the unified formulation for the Frenkel and BMT equations developed in [14]. The model is based on a singular Lagrangian which implies all necessary conditions on spin in a course of the Dirac-Bergmann

\footnotetext{
${ }^{1}$ Note that one needs Hamiltonian to apply Frenkel or BMT-theory for description of Zeeman effect.
} 
algorithm. Besides the position coordinate $x^{\mu}$ and its momentum $p^{\mu}$, our formulation involves an extra vector-like variable $\omega^{\mu}$ with conjugated momentum $\pi^{\mu}$. These can be used to construct both the Frenkel-type spin-tensor and BMT-vector. The Frenkel condition is guaranteed by two constraints ${ }^{2}$ appeared for basic variables, $p \omega=p \pi=0$. The essential point is that the second-class constraints must be taken into account by transition from Poisson to Dirac brackets. As the constraints involve conjugate momenta $p^{\mu}$ for $x^{\mu}$, this leads to nonvanishing brackets for the position variables. As a result, the position space is endowed, in a natural way, with a noncommutative structure which originates from taking into account the spin degrees of freedom. Hence our spinning particle provides an example of noncommutative relativistic system, with noncommutative geometry of position space induced by the spin of the particle. Canonical quantization of the particle gives [17] the one-particle sector of the Dirac equation, so we refer to the particle as Frenkel electron.

The conventional equations of a rotating body in gravitational field (in pole-dipole approximation) were formulated from the analysis of compatibility condition of the energymomentum tensor $[1,2,18]$. These equations, referred to as the Mathisson-Papapetrou (MP) equations, do not form a closed system [2]. Their closure requires an auxiliary Frenkel-type condition on the spin-tensor. Another drawback is that the position coordinate obeys a third-order differential equation $[2,19]$. Nevertheless, since the derivation of the MP equations, they have been applied in different scenarios of general relativity including Schwarzschild and Kerr backgrounds, gravitational waves [20-23] etc. Influence of a curved background on the motion of a gyroscope allows one to test general relativity in a laboratory (nuclear spin gyroscope measurements of the Earth rotation [24]) or orbital experiments (Probe B experiment). Such an influence can be more important for the relativistic regimes [25].

One of the advantages of a variational formulation is in consistent theoretical definition of all variables of a model. Different variational formulations for spinning test particles in gravitational fields exist [26-29]. In [26, 27] the spin-tensor is defined as the conjugated momentum to the angular velocity tensor which is a combination of the tetrad fields and their derivatives. The action is constructed from all invariant quantities that can be formed using the velocity vector and the angular-velocity tensor. In the cited works, supplementary conditions on spin should be added by hands to ensure the correct number of degrees of freedom. In [28], the authors used the formalism of Routhian to reach the MP equations.

The aim of the present work is a two-fold. First, we formulate the variational problem for the Frenkel electron in an arbitrary curved space-time background and study the corresponding equations of motion. Second, we show that in an arbitrary background our equations are compatible with the MP equations, and coincide with them in the weak-field approximation. In other words, the Frenkel electron in a gravitational field has the same behavior as a rotating body in the pole-dipole approximation.

The work is organized as follows. To make it self-contained, in section 2 we give a short review of the Frenkel and BMT approaches and describe the variational formulation

\footnotetext{
${ }^{2} p^{\mu} \sim \dot{x}^{\mu}$ in free theory. In interacting theory the condition $S^{\mu} p_{\mu}=0$ on BMT spin-vector implies $S^{\mu} \dot{x}_{\mu}=0$, see eq. (2.23).
} 
of the Frenkel electron in the presence of an external electromagnetic field. In section 3 we develop a variational formulation of the Frenkel electron in a curved background and study both the Hamiltonian and Lagrangian equations of motion. In section 4 we consider the weak-field limit and compare this with other approaches.

\section{Variational problem for Frenkel and Bargmann-Michel-Telegdi equa- tions}

Frenkel [4] has included the three-dimensional spin-vector $S_{i}$ into the antisymmetric tensor $J^{\mu \nu}=-J^{\nu \mu}$ obeying the constraint

$$
J^{\mu \nu} u_{\nu}=0
$$

where $u_{\nu}=\dot{x}_{\nu}(s)$ represents four-velocity of particle in the proper-time parametrization $s, \dot{x}^{2}(s)=-c^{2}$. In the rest-frame, $u_{\nu}=\left(u_{0}, 0,0,0\right)$, this implies $J^{0 i}=0$. So only three components $J^{i j}=2 \epsilon^{i j k} S^{k}$ of the Frenkel tensor survive. Besides, we can impose the scalar constraint

$$
J^{\mu \nu} J_{\mu \nu}=6 \hbar^{2}
$$

As in the rest frame we have $J^{\mu \nu} J_{\mu \nu}=8\left(S^{i}\right)^{2}$, this implies that we work with spin$1 / 2$ particle.

Frenkel tensor is equivalent to the four-vector

$$
s^{\mu} \equiv \frac{1}{4 \sqrt{-u^{2}}} \epsilon^{\mu \nu \alpha \beta} u_{\nu} J_{\alpha \beta},
$$

which has been taken by Bargmann, Michel and Telegdi as the basic quantity in their description of spin. The BMT-vector of spin obeys the following constraints

$$
s^{\mu} u_{\mu}=0, \quad\left(s^{\mu}\right)^{2}=\frac{3 \hbar^{2}}{4} .
$$

Starting from the non-relativistic precession equation in the rest frame and imposing compatibility with above mentioned constraints we obtain BMT-equations [5]

$$
\begin{aligned}
\ddot{x}^{\mu} & =\frac{e}{m c}(F \dot{x})^{\mu}, \\
\dot{s}^{\mu} & =\frac{e \mu}{m c}(F s)^{\mu}-\frac{e}{m c^{3}}(\mu-1)(s F \dot{x}) \dot{x}^{\mu},
\end{aligned}
$$

for a particle with mass $m$, charge $e$ and magnetic moment $\mu$. In terms of $J_{\mu \nu}$ equation (2.6) reads

$$
\dot{J}^{\mu \nu}=\frac{e}{m c}\left[\mu F^{[\mu}{ }_{\alpha} J^{\alpha \nu]}-\frac{(\mu-1)}{c^{2}}(J F \dot{x})^{[\mu} \dot{x}^{\nu]}\right] .
$$

Let us shortly describe unified variational formulation for both Frenkel and BMT equations $[14,15]$. The basic variables of the model are position $x^{\mu}(\tau)$ and a vector $\omega^{\mu}(\tau)$ taken in arbitrary parametrization $\tau$. Besides, Lagrangian depends on auxiliary variables 
$e_{i}(\tau), i=1,3,4,7$. At the end, they provide all the necessary constraints. Conjugate momenta are denoted as $p^{\mu}, \pi^{\mu}$ and $\pi_{e i}$. Consider the following action

$$
\begin{aligned}
S= & \int d \tau \frac{1}{2 \Delta}\left[e_{3}(\dot{x} N \dot{x})-2 e_{7}(\dot{x} N D \omega)+e_{1}(D \omega N D \omega)\right]+\frac{e}{c} A_{\mu} \dot{x}^{\mu} \\
& -\frac{e_{4}}{2}\left(\omega^{2}-a_{4}\right)-\frac{e_{1}}{2} m^{2} c^{2}+\frac{e_{3}}{2} a_{3},
\end{aligned}
$$

which depends on two numeric parameters $a_{3}$ and $a_{4}$. It has been denoted $\Delta=e_{1} e_{3}-e_{7}^{2}$, $N^{\mu \nu} \equiv \eta^{\mu \nu}-\frac{\omega^{\mu} \omega^{\nu}}{\omega^{2}}$. $F_{\mu \nu}$ is the electromagnetic tensor and $A_{\mu}$ its vector potential. The Lagrangian contains a non-minimal interaction

$$
D \omega^{\mu}=\dot{\omega}^{\mu}-e_{1} \frac{e \mu}{c}(F \omega)^{\mu},
$$

of spin variable $\omega^{\mu}$ with the electromagnetic field. There is no translation symmetry in the space $\omega^{\mu}$, so $\omega^{\mu}$ represents a vector-like variable. According to normalization constraint $\omega^{2}=a_{4}$, the ends of vectors lie on a hyperboloid. We could resolve the constraint and then to omit the term $\frac{e_{4}}{2}\left(\omega^{2}-a_{4}\right)$ from the action, but this lead to lose of manifest relativistic covariance of the formalism. Besides, we point out that the vectors $\omega^{\mu}$ change under spinplane local symmetry. For detailed analysis of resulting phase-space geometry see [30]. In short, in non-relativistic models of spin, inner manifold which describes the spin degrees of freedom is two sphere. The constraint $\omega^{2}=a_{4}$ represents a covariant generalization of this construction. It can be seen by transition to the rest frame for the free particle. In this frame $\omega^{0}=0$, and spacial components $\vec{\omega}$ belong to the sphere $\vec{\omega}^{2}=a_{4}$.

The action is invariant under two-parametric group of local symmetries which consist of reparametrizations and spin-plane transformations, see [31] for details. As we deal with a singular Lagrangian, the Dirac-Bergmann algorithm is necessary to study the model in Hamiltonian formulation. Performing transition to the Hamiltonian formulation, we find the following constraints

$$
\begin{gathered}
\mathcal{P}^{2}-\frac{\mu e}{2 c} F^{\mu \nu} J_{\mu \nu}+m^{2} c^{2}=0, \\
\pi^{2}-a_{3}=0, \quad \omega^{2}-a_{4}=0, \quad \omega \pi=0, \\
\mathcal{P} \omega=0, \quad \mathcal{P} \pi=0 .
\end{gathered}
$$

The equation (2.10) is the standard mass-shell condition [32,33]. This turns out to be the first-class constraint. $\mathcal{P}_{\mu}$ is defined in terms of conjugate momenta $p_{\mu}$ and the electromagnetic vector potential as follows

$$
\mathcal{P}^{\mu}=p^{\mu}-\frac{e}{c} A^{\mu}
$$

The relation with Frenkel theory is reached by defining the Frenkel-type spin-tensor

$$
J^{\mu \nu}=2\left(\omega^{\mu} \pi^{\nu}-\omega^{\nu} \pi^{\mu}\right) .
$$

Then eqs. (2.12) guarantee its transversality

$$
J^{\mu \nu} \mathcal{P}_{\nu}=0
$$


while eqs. (2.11) tell us that

$$
J^{\mu \nu} J_{\mu \nu}=8\left(\omega^{2} \pi^{2}-(\omega \pi)^{2}\right)=8 a_{3} a_{4} .
$$

This fixes the value of spin by an appropriate choice of the numbers $a_{3}$ and $a_{4}$.

The set (2.11), (2.12) contains one first-class constraint. Taking into account that each second-class constraint rules out one phase-space variable, whereas each first-class constraint rules out two variables, we have the right number of spin degrees of freedom, $8-(4+2)=2$.

For the case of an arbitrary background, the action leads to rather complicated equations of motion, see [15]. So we present here their form in proper-time parametrization, for uniform electromagnetic field

$$
\begin{aligned}
\frac{d(\tilde{T} \dot{x})^{\mu}}{d \tau} & =\frac{e}{m_{r} c}(F \dot{x})^{\mu}, \\
\dot{J}^{\mu \nu} & =\frac{e}{m_{r} c}\left[\mu F^{[\mu}{ }_{\alpha} J^{\alpha \nu]}-\frac{m_{r}^{2}(\mu-1)}{c^{2} M^{2}}(J F \tilde{T} \dot{x})^{[\mu}(\tilde{T} \dot{x})^{\nu]}\right], \\
\dot{s}^{\mu} & =\frac{e \mu}{m_{r} c}(F s)^{\mu}-\frac{e}{m_{r} c^{3}}[(\mu-1)(s F \dot{x})+\mu b(s F J F \dot{x})](\tilde{T} \dot{x})^{\mu} .
\end{aligned}
$$

The following notation were used

$$
\begin{aligned}
\tilde{T}^{\mu \nu} & \equiv \eta^{\mu \nu}-\frac{2 e(\mu-1)}{2 m^{2} c^{3}\left(3 \Delta^{2}-1\right)}(J F)^{\mu \nu}, & \Delta^{2} & \equiv 1-\frac{\mu e}{2 m^{2} c^{3}}(J F), \\
m_{r}^{2} & =m^{2}-\frac{\mu e}{2 c^{3}}(J F), & M^{2} & =m^{2}-\frac{e(2 \mu+1)}{4 c^{3}}(J F) .
\end{aligned}
$$

These equations are written in an arbitrary parametrization of the world-line. In (2.19) the spin vector if defined by

$$
s^{\mu} \equiv \frac{1}{4 \sqrt{-\mathcal{P}^{2}}} \epsilon^{\mu \nu \alpha \beta} \mathcal{P}_{\nu} J_{\alpha \beta}
$$

By construction, $s^{\mu}$ obeys

$$
s^{\mu} \mathcal{P}_{\mu}=s^{\mu} \dot{x}_{\mu}=0, \quad s_{\mu} s^{\mu}=a_{3} a_{4} .
$$

The BMT equations (2.5) and (2.7) appeared neglecting higher-order terms of $\hbar$ in equations (2.17) and (2.19). In the case of a free particle, $F^{\mu \nu}=0$, both spin-tensor and spin-vector are conserved quantities, $\dot{J}^{\mu \nu}=0, \quad \dot{s}^{\mu}=0$.

\section{Spinning particle in a curved space-time}

We obtain variational formulation of Frenkel particle in a curved background by writing the action (2.8) with $A^{\mu}=0$ in a generally covariant form. It is sufficient to replace the Minkowski metric $\eta_{\mu \nu}$ by an arbitrary metric $g_{\mu \nu}\left(x^{\rho}\right)$, and usual derivative of contravariant vector $\omega^{\mu}$ by the covariant derivative

$$
\dot{\omega}^{\mu} \rightarrow D \omega^{\mu}=\dot{\omega}^{\mu}+\Gamma_{\alpha \beta}^{\mu} \dot{x}^{\alpha} \omega^{\beta} .
$$


Then the action reads

$$
\begin{aligned}
S_{g}= & \int d \tau \frac{1}{2 \Delta}\left[e_{3}(\dot{x} N \dot{x})-2 e_{7}(\dot{x} N D \omega)+e_{1}(D \omega N D \omega)\right]-\frac{e_{4}}{2}\left(\omega^{2}-a_{4}\right) \\
& -\frac{e_{1}}{2} m^{2} c^{2}+\frac{e_{3}}{2} a_{3} .
\end{aligned}
$$

Velocities $\dot{x}^{\mu}, D \omega^{\mu}$ and projector $N^{\mu \nu}=g^{\mu \nu}-\frac{\omega^{\mu} \omega^{\nu}}{\omega^{2}}$ transform as contravariant tensors, therefore the Lagrangian is manifestly invariant under general coordinate transformations. In the flat limit, $g_{\mu \nu}=\eta_{\mu \nu}$, the action (3.2) reduces to (2.8) with $A^{\mu}=0$.

\subsection{Hamiltonian constraints}

Let us construct Hamiltonian formulation of the theory (3.2). The conjugate momenta of $\omega^{\mu}$ and $x^{\mu}$ are

$$
\begin{aligned}
\pi_{\mu} & =-\frac{e_{7}}{\Delta} N_{\mu \nu} \dot{x}^{\nu}+\frac{e_{1}}{\Delta_{e}} N_{\mu \nu} D \omega^{\nu} \\
p_{\mu} & =\frac{e_{3}}{\Delta} N_{\mu \nu} \dot{x}^{\nu}-\frac{e_{7}}{\Delta_{g}} N_{\mu \nu} D \omega^{\nu}+\Gamma_{\mu \nu}^{\alpha} \omega^{\nu} \pi_{\alpha} .
\end{aligned}
$$

According to this expression, $\pi_{\mu}$ is a covariant vector. Concerning the second line, due to the presence of Christoffel symbols, $p_{\mu}$ does not transform as a vector. Using transformation law of $\Gamma_{\mu \nu}^{\alpha}$ and the fact that other quantities transform as vectors, we get transformation law of $p^{\mu}$

$$
p_{\mu}^{\prime}=\frac{\partial x^{\alpha}}{\partial x^{\prime \mu}} p_{\alpha}+\frac{\partial x^{\prime \nu}}{\partial x^{\beta}} \frac{\partial^{2} x^{\sigma}}{\partial x^{\prime \mu} \partial x^{\prime \nu}} \omega^{\beta} \pi_{\sigma} .
$$

Eq. (3.4) prompts to introduce the covariant momentum $P_{\mu}$ which transforms as a vector

$$
P_{\mu}=p_{\mu}-\Gamma_{\mu \beta}^{\alpha} \omega^{\beta} \pi_{\alpha}
$$

The projector $N^{\mu \nu}$ has null vector $\omega_{\mu}$, therefore equations (3.3) and (3.4) imply the primary constraints

$$
\begin{aligned}
& T_{5} \equiv \omega^{\mu} \pi_{\mu}=0, \\
& T_{6} \equiv \omega^{\mu} P_{\mu}=0 .
\end{aligned}
$$

Conjugate momenta of $e_{i}$ vanish, $\pi_{e i}=0$, therefore we get four more primary constraints. Applying the Legendre transformation to (3.2) and using (3.3), (3.4), we obtain the following Hamiltonian

$$
\begin{aligned}
H= & \frac{1}{2} e_{1}\left(g^{\mu \nu} P_{\mu} P_{\nu}+m^{2} c^{2}\right)+\frac{1}{2} e_{3}\left(g^{\mu \nu} \pi_{\mu} \pi_{\nu}-a_{3}\right) \\
& +\frac{1}{2} e_{4}\left(g_{\mu \nu} \omega^{\mu} \omega^{\nu}-a_{4}\right)+\lambda_{5} \pi_{\mu} \omega^{\mu}+e_{7} g^{\mu \nu} P_{\mu} \pi_{\nu}+\lambda_{6} P_{\mu} \omega^{\mu}+\lambda_{e i} \pi_{e i}
\end{aligned}
$$

where $\lambda_{5}$ and $\lambda_{6}$ appear as lagrangian multipliers for primary constraints $T_{5}$ and $T_{6}$, respectively, and $\lambda_{e i}$ stand for Lagrangian multipliers associated with the primary constraints $\pi_{e i}$. 
The Hamiltonian (3.8) consist of covariant quantities only, hence it is manifestly invariant under general-coordinate transformations. Following the Dirac procedure for singular systems we conclude that

$$
\begin{array}{ll}
T_{1}=g^{\mu \nu} P_{\mu} P_{\nu}+m^{2} c^{2}=0, & T_{3}=g^{\mu \nu} \pi_{\mu} \pi_{\nu}-a_{3}=0, \\
T_{4}=g_{\mu \nu} \omega^{\mu} \omega^{\nu}-a_{4}=0, & T_{7}=g^{\mu \nu} P_{\mu} \pi_{\nu}=0,
\end{array}
$$

appear as secondary constraints when we impose the compatibility conditions $\dot{\pi}_{e i}=0$. The second, third and fourth stages of the Dirac-Bergmann algorithm can be resumed as follows

$$
\begin{aligned}
& T_{1}=0 \quad \Rightarrow \quad \lambda_{6} R_{(\omega)}+e_{7} R_{(\pi)}=0, \\
& T_{3}=0 \quad \Rightarrow \quad \lambda_{5}=0, \\
& T_{4}=0 \quad \Rightarrow \quad \lambda_{5}=0, \\
& T_{5}=0 \quad \Rightarrow \quad a_{3} e_{3}-a_{4} e_{4}=0, \quad \Rightarrow \quad \lambda_{e_{4}}=\frac{a_{3}}{a_{4}} \lambda_{e 3}, \\
& T_{6}=0 \quad \Rightarrow \quad e_{1} R_{(\omega)}+2 e_{7} M^{2}=0, \quad \Rightarrow \quad \lambda_{e 7}=f\left(\lambda_{e 1}\right), \\
& T_{7}=0 \quad \Rightarrow \quad e_{1} R_{(\pi)}-2 \lambda_{6} M^{2}=0,
\end{aligned}
$$

where we defined the scalar quantities

$$
\begin{aligned}
R_{(\pi)} & \equiv 2 R_{\alpha \beta \mu \nu} \pi^{\alpha} \omega^{\beta} \pi^{\mu} P^{\nu}, \quad R_{(\omega)} \equiv 2 R_{\alpha \beta \mu \nu} \pi^{\alpha} \omega^{\beta} \omega^{\mu} P^{\nu} \\
M^{2} & \equiv m^{2} c^{2}-R_{\alpha \mu \beta \nu} \omega^{\alpha} \pi^{\mu} \omega^{\beta} \pi^{\nu} .
\end{aligned}
$$

Here $R_{\mu \nu \alpha \beta}$ is the curvature tensor. Eq. (3.10) turns out to be a consequence of (3.14) and (3.15) and can be omitted. Eq. (3.14) determines $e_{7}=-\frac{R_{(\pi)}}{2 M^{2}} e_{1}$ while (3.15) gives the lagrangian multiplier $\lambda_{6}=\frac{R_{(\pi)}}{2 M^{2}} e_{1}$. The Dirac-Bergmann algorithm stops at the fourth stage and results in six non-trivial constraints $T_{a}, a=1,3,4,5,6,7$. The multipliers $\lambda_{e 1}, \lambda_{e 3}$ and the variables $e_{1}, e_{3}$ have not been determined in the process. This is in agreement with invariance of our action with respect to two local symmetries mentioned above. Poisson brackets of constraints are shown in table 1.

The Hamiltonian (3.8) turns out to be a combination of constraints

$$
H=\frac{1}{2} e_{1}\left[T_{1}+\frac{R_{(\pi)}}{M^{2}} T_{6}-\frac{R_{(\omega)}}{M^{2}} T_{7}\right]+\frac{1}{2} e_{3}\left[T_{3}+\left(a_{3} / a_{4}\right) T_{4}\right]+\lambda_{e i} \pi_{e i} .
$$

The constraint matrix $\Delta_{a b} \equiv\left\{T_{a}, T_{b}\right\}$, which can be easily determinate from the table 1 , has $\operatorname{rank}\left[T_{a b}\right]=4$. Its null-vectors are $\zeta_{1}=\left(M^{2}, 0,0,0, R_{(\pi)},-R_{(\omega)}\right)$ and $\zeta_{2}=$ $\left(0, a_{4} / a_{3}, 1,0,0,0\right)$. They determine two first-class constraints of the model

$$
\Psi_{1}=T_{1}+\frac{R_{(\pi)}}{M^{2}} T_{6}-\frac{R_{(\omega)}}{M^{2}} T_{7}, \quad \Psi_{2}=T_{3}+\left(a_{3} / a_{4}\right) T_{4}
$$

As a consequence, the Hamiltonian (3.18) represents a first-class quantity. Adding four linearly-independent vectors $\zeta_{3}=(0,1,0,0,0,0), \quad \zeta_{4}=(0,0,0,1,0,0), \quad \zeta_{5}=(0,0,0,0,1,0)$ 


\begin{tabular}{|c|c|c|c|c|c|c|}
\hline & $T_{1}$ & $T_{3}$ & $T_{4}$ & $T_{5}$ & $T_{6}$ & $T_{7}$ \\
\hline$T_{1}=P^{2}+m^{2} c^{2}$ & 0 & 0 & 0 & 0 & $R_{(\omega)}$ & $R_{(\pi)}$ \\
\hline$T_{3}=\pi^{2}-a_{3}$ & 0 & 0 & $-4 T_{5}$ & $2\left(T_{3}-a_{3}\right)$ & $-2 T_{7}$ & 0 \\
\hline$T_{4}=\omega^{2}-a_{4}$ & 0 & $4 T_{5}$ & 0 & $2\left(a_{4}-T_{4}\right)$ & 0 & $2 T_{6}$ \\
\hline$T_{5}=\omega \pi$ & 0 & $2\left(a_{3}-T_{3}\right)$ & $2\left(T_{4}-a_{4}\right)$ & 0 & $-T_{6}$ & $T_{7}$ \\
\hline$T_{6}=P \omega$ & $-R_{(\omega)}$ & $2 T_{7}$ & 0 & $T_{6}$ & 0 & $-M^{2}$ \\
\hline$T_{7}=P \pi$ & $-R_{(\pi)}$ & 0 & $-2 T_{6}$ & $-T_{7}$ & $M^{2}$ & 0 \\
\hline
\end{tabular}

Table 1. Algebra of constraints.

and $\zeta_{6}=(0,0,0,0,0,1)$ to $\zeta_{1}$ and $\zeta_{2}$, we construct an equivalent system of constraints, $\left\{\Psi_{A}=\zeta \cdot T\right\}$. They read $\Psi_{3}=T_{3}, \Psi_{4}=T_{5}, \Psi_{5}=T_{6}, \Psi_{6}=T_{7}$. In the set $\left\{\Psi_{A}\right\}$, constraints $\Psi_{1}$ and $\Psi_{2}$ form first-class subset while $\Psi_{3}, \ldots, \Psi_{6}$ form a second-class system with non-degenerate constraint-matrix $\Delta_{a b}=\left\{\Psi_{a}, \Psi_{b}\right\}$. Its inverse reads

$$
\Delta^{a b}=\left(\begin{array}{cccc}
0 & 1 / 2 a_{3} & 0 & 0 \\
-1 / 2 a_{3} & 0 & 0 & 0 \\
0 & 0 & 0 & 1 / M^{2} \\
0 & 0 & -1 / M^{2} & 0
\end{array}\right) .
$$

This matrix determines Dirac bracket constructed on the base of second-class constraints

$$
\{A, B\}_{D}=\{A, B\}-\left\{A, \Psi_{a}\right\} \Delta^{a b}\left\{\Psi_{b}, B\right\}, \quad a, b=3,4,5,6 .
$$

Passing to the Dirac bracket, we can work with much more simple Hamiltonian, which does not involve an auxiliary variables, see eq. (4.12) below. We construct the Dirac bracket (see appendix A) and note that our position variables obey highly noncommutative algebra

$$
\left\{x^{\mu}, x^{\nu}\right\}_{D}=\frac{J^{\mu \nu}}{2 M^{2}} .
$$

We can pass from parametric $x^{\mu}(\tau)$ to physical variables $x^{i}(t)$. As a consequence of (3.22), $x^{i}(t)$ also obey a noncommutative algebra.

Let us discuss this point in some details. In classical mechanics without constraints, the initial variables usually have an interpretation as the position variables and obey the Poisson bracket. This situation may change in a theory with constraints. In a theory with second-class constraints one can find special coordinates on the constraints surface with canonical (that is Poisson) bracket [34]. Functions of special coordinates are candidates for observable quantities. The Dirac bracket (more exactly, its nondegenerated part) is just the canonical bracket rewritten in terms of initial variables. For the present case, namely the initial coordinates which correspond to non-degenerated sector (they are $x^{i}(t)$ ) are of physical interest, as they represent the position of a particle. So, while there are special coordinates with canonical symplectic structure, the physically interesting coordinates obey non-commutative algebra (3.22). Remind that a theory with second-class constraints 
can not be consistently quantized on the base of Poisson bracket. We need to postulate commutators that resemble the Dirac bracket. So the position variables will be presented by non commutative operators in quantum theory, see [17].

In summary, to incorporate the Frenkel condition $J^{\mu \nu} P_{\mu}=0$ in a proper way, we need a model with two second-class constraints, $P \omega=P \pi=0$, which involve conjugate momenta of $x^{\mu}$. As a consequence, $x^{\mu}$ have nonvanishing Dirac brackets and the position space is endowed, in a natural way, with noncommutative geometry. Our description of spin on the base of vector variables gives one more example of physically interesting noncommutative relativistic particle, with the "parameter of noncommutativity" proportional to spin-tensor. There are other examples where the noncommutative geometry emerges from second-class constraints, see [35-44].

We have demonstrated that our spinning particle admits interaction with general curved background, this does not break neither the number nor the structure of constraints presented in the free theory. Having established the structure of our model in Hamiltonian formulation, we proceed further analysis at the level of equations of motion.

\section{Hamiltonian and Lagrangian equations of motion in curved background}

The dynamics of basic variables is governed by Hamiltonian equations $\dot{z}=\{z, H\}, z=$ $(x, p, \omega, \pi)$, with the Hamiltonian (3.18). After some algebra, these equations can be written in a manifestly covariant form

$$
\begin{aligned}
\dot{x}^{\mu} & =e_{1}\left[P^{\mu}+\left(2 M^{2}\right)^{-1}\left(R_{(\pi)} \omega^{\mu}-R_{(\omega)} \pi^{\mu}\right)\right] \\
D P_{\mu} & =R_{\beta \nu \mu}^{\alpha} \pi_{\alpha} \omega^{\beta} \dot{x}^{\nu}, \\
D \omega^{\mu} & =-e_{1} \frac{R_{(\omega)}}{2 M^{2}} P^{\mu}+e_{3} \pi^{\mu}, \quad D \pi_{\mu}=-e_{1} \frac{R_{(\pi)}}{2 M^{2}} P_{\mu}-e_{3}\left(a_{3} / a_{4}\right) \omega_{\mu} .
\end{aligned}
$$

Due to presence of arbitrary functions $e_{1}$ and $e_{3}$, the evolution of our basic variables is ambiguous. This is in correspondence with two local symmetries presented in the theory. Position $x^{\mu}$ and momentum $P_{\mu}$ have one-parametric ambiguity due to $e_{1}$. Basic spin variables $\omega^{\mu}$ and $\pi_{\mu}$ have two-parametric ambiguity due $e_{1}$ and $e_{3}$. According to general theory [34, 45], variables with ambiguous dynamics do not represent observable quantities. So let us look for the variables that can be candidates for observables. Equation of motion for the spin-tensor

$$
J^{\mu \nu} \equiv 2\left(\omega^{\mu} \pi^{\nu}-\omega^{\nu} \pi^{\mu}\right),
$$

does not contain $e_{3}$ (see below). According to geometric construction described in [31], the quantities $J^{\mu \nu}$, being functions of coordinates of the base of spin fiber-bundle, are inert under the spin-plane local symmetry. By construction, on the constraint surface (3.7) and (3.9) the spin-tensor obeys the conditions

$$
\begin{aligned}
J^{\mu \nu} P_{\nu} & =0, \\
J^{\mu \nu} J_{\mu \nu} & =8 a_{3} a_{4} .
\end{aligned}
$$


We mention that eq. (4.5) usually imposed by hand in the study of a test-body motion in general relativity in pole-dipole approximation [20-23]. In our approach this condition is embedded into the model from the beginning.

Let us write Hamiltonian equations for $x, P$ and $J$. To exclude $\omega$ and $\pi$ from (4.1), we use (4.4) presenting the quantities defined in (3.16) and (3.17) as functions of spin-tensor

$$
R_{(\pi)}=\frac{1}{2} R_{\alpha \beta \mu \nu} J^{\alpha \beta} P^{\mu} \pi^{\nu}, \quad R_{(\omega)}=\frac{1}{2} R_{\alpha \beta \mu \nu} J^{\alpha \beta} P^{\mu} \omega^{\nu}, \quad M^{2}=m^{2} c^{2}-\frac{1}{16} \theta_{\mu \nu} J^{\mu \nu} .
$$

Tensor $\theta_{\mu \nu}$ is defined as follows

$$
\theta_{\mu \nu} \equiv R_{\alpha \beta \mu \nu} J^{\alpha \beta}
$$

With this, Hamiltonian equation for the coordinate $x^{\mu}$ can be written in terms of the momentum $P_{\mu}$ and spin-tensor $J^{\mu \nu}$. The eqs. (4.4) and (4.3) immediately imply an equation of motion for $J^{\mu \nu}$. Using symmetry properties of the curvature tensor, equation (4.2) can be written in terms of $J$ and $\dot{x}$. In the result, Hamiltonian equations can be written only in terms of the variables $x, P$ and $J$

$$
\begin{aligned}
\dot{x}^{\mu} & =e_{1}\left[P^{\mu}-\frac{1}{8 M^{2}} P^{\sigma} \theta_{\sigma \nu} J^{\nu \mu}\right], \\
D P_{\mu} & =\frac{1}{4} R_{\mu \nu \alpha \beta} \dot{x}^{\nu} J^{\alpha \beta}, \\
D J^{\mu \nu} & =2\left[P^{\mu} \dot{x}^{\nu}-P^{\nu} \dot{x}^{\mu}\right] .
\end{aligned}
$$

Together with (4.5) and (4.6), they form closed system which determines evolution of a spinning particle. The remaining ambiguity due to $e_{1}$ is related with reparametrization invariance, and can be removed in the standard way, passing to the physical-time parametrization. Hence one possible set of candidates for observables is $\left(x^{i}(t), P^{i}(t), J^{\mu \nu}(t)\right)$.

Hamiltonian can also be rewritten in terms of $x, P$ and $J$. We note that $\pi^{2}, \omega^{2}$ and $\omega \pi$, being $\mathrm{SO}(1,3)$-invariants, have vanishing brackets with $J^{\mu \nu}$. As a consequence, the second, third, fourth and the last terms in (3.8) do not contribute into equations (4.9)-(4.11). So we omit these terms in the Hamiltonian. Further, supposing that we deal with the Dirac bracket, we can use the constraints $T_{6}$ and $T_{7}$ in (3.8). Then the terms proportional to $e_{7}$ and $\lambda_{6}$ vanish. In the result, the Hamiltonian reads

$$
H_{1}=\frac{1}{2} e_{1}\left(g^{\mu \nu} P_{\mu} P_{\nu}+m^{2} c^{2}\right) .
$$

This produces the equations (4.9)-(4.11) as follows: $\dot{q}=\left\{q, H_{1}\right\}_{D}$.

As it should be expected, they imply second-order equation for the position of a particle. Equations (4.10) and (4.11) coincide with those of Papapetrou [2, 25]. The r.h.s. of equation (4.10) points out an influence of spin on the trajectory and can be interpreted as a spin-orbit interaction. Note that this originates from second term on r.h.s. of the covariant derivative (3.1). In turn, this term is necessary to provide general covariance of the formalism.

Besides, eq. (4.11) implies the equation

$$
D J^{\mu \nu}+\frac{1}{m^{2}} P^{\nu} P_{\alpha} D J^{\mu \alpha}-\frac{1}{m^{2}} P^{\mu} P_{\alpha} D J^{\nu \alpha}=0,
$$

which has been taken by Papapetrou as the basic equation for spin, see his equation (5.3). 
In the next section we show that (4.9)-(4.11) coincide with those of Papapetrou in the leading-spin limit.

Eliminating $P_{\mu}$ and $e_{1}$ from the system (4.9), (4.11), we obtain its Lagrangian form (for the sector $x$ ). We first note that the relation between velocity and momentum (4.9) can be written as a linear transformation

$$
\dot{x}^{\mu}=e_{1} T_{\nu}^{\mu} P^{\nu}, \quad T^{\mu}{ }_{\nu} \equiv \delta_{\nu}^{\mu}-\frac{1}{8 M^{2}} J^{\mu \alpha} \theta_{\alpha \nu} .
$$

Using the definition of the spin-tensor (4.4), we find the identity

$$
J^{\mu \alpha} \theta_{\alpha \sigma} J^{\sigma \beta} \theta_{\beta \nu}=\frac{1}{2}(J \cdot \theta) J^{\mu \alpha} \theta_{\alpha \nu},
$$

where the term $J \cdot \theta=J^{\mu \nu} \theta_{\mu \nu}$ is proportional to the gravity-magnetic moment interaction [46]. The identity allows us to find the inverse matrix

$$
\left(T^{-1}\right)^{\mu}{ }_{\nu}=\delta_{\nu}^{\mu}+\frac{J^{\mu \alpha} \theta_{\alpha \nu}}{8 m^{2} c^{2}-J \cdot \theta} .
$$

Then eq. (4.14) can be inverted, and substituting $P^{\mu}=P^{\mu}\left(\dot{x}, J, e_{1}\right)$ into expression for the constraint $T_{1}, g^{\mu \nu} P_{\mu} P_{\nu}+m^{2} c^{2}=0$, this gives $e_{1}$

$$
e_{1}=\frac{\sqrt{-G_{\mu \nu} \dot{x}^{\mu} \dot{x}^{\nu}}}{m c} \equiv \frac{\sqrt{-\dot{x} G \dot{x}}}{m c}
$$

We have introduced the effective metric

$$
G_{\mu \nu} \equiv\left(T^{-1}\right)^{\alpha}{ }_{\mu} g_{\alpha \beta}\left(T^{-1}\right)^{\beta}{ }_{\nu}
$$

The momentum (3.6), (3.4) acquires the following form:

$$
P^{\mu}=\frac{m c}{\sqrt{-\dot{x} G \dot{x}}}\left(T^{-1}\right)^{\mu}{ }_{\nu} \dot{x}^{\nu}=\frac{m c}{\sqrt{-\dot{x} G \dot{x}}}\left[\dot{x}^{\mu}+\frac{1}{8 m^{2} c^{2}-J \cdot \theta} R_{\nu \sigma \alpha \beta} J^{\mu \nu} J^{\alpha \beta} \dot{x}^{\sigma}\right] .
$$

Similar expression for $P^{\mu}$ has been obtained in [27] as a compatibility condition of Papapetrou equations (4.10) and (4.11) with the thansversality condition. Unlike them our momentum $P_{\mu}$ is defined through the standard Hamiltonian formalism. Besides, our spintensor automatically obeys the Frenkel condition while in [27] the author must demand both (4.5) and its conservation by hands.

Substituting (4.19) into (4.10) we get the Lagrangian equation for $x^{\mu}$

$$
D\left[\frac{\left(T^{-1}\right)^{\mu}{ }_{\nu} \dot{x}^{\nu}}{\sqrt{-\dot{x} G \dot{x}}}\right]=\frac{1}{4 m c} R_{\nu \alpha \beta}^{\mu} \dot{x}^{\nu} J^{\alpha \beta} .
$$

Similarly, removing $P^{\mu}$ from (4.11) we obtain

$$
D J^{\mu \nu}=\frac{2 m c}{\sqrt{-\dot{x} G \dot{x}}}\left[\left(T^{-1}\right)^{[\mu}{ }_{\alpha} \dot{x}^{\nu]} \dot{x}^{\alpha}\right] .
$$

These equations together (4.5) and (4.6) form closed system for the set $x, J$. 


\section{$5 \quad$ First order approximation}

If we study a particle like the electron, then $J^{2} \sim \hbar^{2}$. At the end of the section 2 we mentioned that in the limit $\hbar^{2} \ll 1$ our equations for an electron in uniform electromagnetic field reduce to those of BMT. In this section we consider a similar approximation, $J^{2} / c^{2} \ll$ 1 , for our particle in gravitational fields. In such approximation the matrix $T^{-1}$ in (4.16) reduces to the identity matrix and the effective metric $G_{\mu \nu}$ coincides with the initial metric tensor. The equation (4.20) is simplified

$$
D\left[\frac{\dot{x}^{\mu}}{\sqrt{-\dot{x} g \dot{x}}}\right]=\frac{1}{4 m c} R_{\nu \alpha \beta}^{\mu} \dot{x}^{\nu} J^{\alpha \beta} .
$$

In (4.21) the r.h.s. vanishes

$$
D J^{\mu \nu}=0 .
$$

The conditions (4.5) and (4.6) read as follows: $J^{\mu \nu} \dot{x}_{\nu}=0, J^{\mu \nu} J_{\mu \nu}=8 a_{3} a_{4} \ll c^{2}$. The equation (5.1) coincides with the MP equation neglecting the terms nonlinear in spin [46]. Using the Levi-Civita symbol we define the spin vector

$$
s^{\mu} \equiv \frac{g^{-1 / 2}}{4 \sqrt{-\dot{x} g \dot{x}}} \varepsilon^{\mu \nu \alpha \beta} \dot{x}_{\nu} J_{\alpha \beta} .
$$

Our approximation implies simple equation for $s^{\mu}$. To simplify the notation it is convenient to chose the proper-time parametrization $s$. The equation for the trajectory of the test particle now reads

$$
D_{s} u_{\mu}=\frac{g^{-1 / 2}}{2 m c} R_{\mu \nu \alpha \beta} \varepsilon^{\alpha \beta \sigma \kappa} s_{\sigma} u_{\kappa} u^{\nu},
$$

where $u_{\mu} \equiv\left(d x_{\mu} / d s\right)$. Defining

$$
F_{\mu \nu} \equiv \frac{g^{-1 / 2}}{2} R_{\mu \nu \alpha \beta} \varepsilon^{\alpha \beta \sigma \kappa} s_{\sigma} u_{\kappa}
$$

equation (5.4) can be written in terms of effective Lorentz force

$$
D_{s} u_{\mu}=\frac{1}{m c} F_{\mu \nu} u^{\nu}
$$

Equation (4.21) and definition (5.3) imply that

$$
D_{s} s^{\mu}=0
$$

where we have taken into account that $F_{\mu \nu}$ is of first order in $S^{\mu}(\sim \hbar$ for the electron). The supplementary spin conditions for $s^{\mu}$ are

$$
s^{\mu} u_{\mu}=0, \quad s^{\mu} s_{\mu}=a_{3} a_{4} \ll c^{2} .
$$

Equations (5.6), (5.7) and (5.8) determine behavior of both trajectory and spin in a gravitational field. 
Let us consider the weak-field approximation

$$
g_{\mu \nu}=\eta_{\mu \nu}+h_{\mu \nu}, \quad\left|h_{\mu \nu}\right| \ll 1,
$$

and a spherically symmetric field produced by a mass $M_{s}$

$$
h_{00}=\frac{2 k M_{s}}{c^{2} r}, \quad h_{i j}=-\frac{2 k M_{s}}{c^{2} r} \delta_{i j}, \quad h_{0 i}=0 .
$$

The corresponding equation of motion for a test spinning particle derived from eq. (5.1) (or, alternatively, form (5.6)) is

$$
\ddot{\mathbf{x}}=-\frac{k M_{s}}{c^{2}} \frac{\mathbf{x}}{x^{3}}-3 \frac{k M_{s}}{m c^{2}} \frac{1}{x^{3}}[[\dot{\mathbf{x}} \times \mathbf{S}]-(\hat{\mathbf{n}} \cdot \dot{\mathbf{x}})[\hat{\mathbf{n}} \times \mathbf{S}]-2([\dot{\mathbf{x}} \times \mathbf{S}] \cdot \hat{\mathbf{n}}) \hat{\mathbf{n}}] .
$$

Here $\hat{\mathbf{n}}=\mathbf{x} / x$ and $\mathbf{S}$ is the spacial part of spin vector (5.3), $M_{s}$ is the mass of the source of the gravitational field and $m$ is the mass of the spinning test particle. The equation coincides with the MP equation taken in the weak field approximation, see [47]. Hence, in all practically interesting cases, evolution of the Frenkel particle in a gravitational background coincides with that of a rotating body in pole-dipole approximation.

The acceleration (5.11) is different from that obtained in the quantum theory of Dirac equation $[48,49]$

$$
\ddot{\mathbf{r}}=-\frac{k M_{s}}{c^{2}} \frac{\mathbf{r}}{r^{3}}-3 \frac{k M_{s}}{m c^{2}} \frac{1}{r^{3}}\left([\dot{\mathbf{r}} \times \mathbf{S}]-\frac{3}{2}(\mathbf{n} \cdot \dot{\mathbf{r}})[\mathbf{n} \times \mathbf{S}]-\frac{3}{2}([\dot{\mathbf{r}} \times \mathbf{S}] \cdot \mathbf{n}) \mathbf{n}\right),
$$

where $\mathbf{n}=\mathbf{r} / r$. The last equation also can be obtained if one starts with classical mechanics of two spinning particles with spin-orbit interaction [46].

One possible explanation of the difference between (5.11) and (5.12) has been suggested in $[19,46,47]$. For the case of a rotating object, one can adopt different definitions of its center-of-mass. If we relate the variables $\mathbf{x}$ and $\mathbf{r}$ (in the considered approximation) as follows:

$$
\mathbf{x}=\mathbf{r}+\frac{1}{m c}[\dot{\mathbf{r}} \times \mathbf{S}]
$$

the equation (5.11) turn into (5.12). So the discrepancy may be explained as coming from different definitions of the center of mass for a rotating object.

Let us point out another explanation. In a number of classical-mechanical models of Dirac equation $[13,16,50,51]$, the variable $\mathbf{r}$, which corresponds to the position operator $\hat{\mathbf{r}}$ of the Dirac equation, is a gauge non-invariant variable. So $\mathbf{r}$ is non-observable quantity. The observable quantity $\mathbf{x}$ turns out to be related with $\mathbf{r}$ just according the equation (5.13).

Concerning the equation (5.13), we also point out that in the approximation under consideration, the Dirac brackets (3.21) imply $\left\{r^{i}, r^{j}\right\}_{D}=0$, that is the radius-vector in eq. (5.12) corresponds to the canonical coordinates of our theory, see discussion at the end of section 3 . 


\section{Conclusions}

In this work we have extended the variational formulation of a spinning particle in an electromagnetic field [14] to the case of a curved space-time background. This demonstrates an effectiveness and generality of the classical description of spin on the base of vector-like variable. Our model allows interaction with an arbitrary curved background without modifications of the number and the structure of constraints (2.10)-(2.12). The supplementary spin conditions (4.5), (4.6) are guaranteed by the constraints (2.11) and (2.12) arising from our singular Lagrangian (3.2).

We derived a closed system of Hamilton equations (4.9)-(4.11) for the position, momentum and spin-tensor. If we work with Dirac brackets, these equations can be obtained as Hamiltonian equations with a simple Hamiltonian written in eq. (4.12). As expected, position coordinates obeys the second-order equation, while the equations (4.10) and (4.11) are compatible with those of Papapetrou [2]. In the practically important case of the leading-spin approximation our equations coincide with those of Papapetrou.

Excluding the momenta $P^{\mu}$ from the Hamiltonian equations, we obtained their Lagrangian form in terms of $x^{\mu}$ and $J^{\mu \nu}$, see equations (4.20) and (4.21). Linear in spin approximation of these equations yields a simple equation for the spin-vector $S^{\mu}$. Combining this with the weak-field approximation used in general relativity, we get equations of motion for a spinning object in a gravitational field with spherical symmetry. We hope that the developed variational model will be useful for the description and analysis of experimental setups involving relativistic spinning particles in a gravitational background.

It is known that the formalism of dynamical systems with second-class constraints implies a natural possibility to incorporate noncommutative geometry into the framework of classical and quantum theory [35, 36]. Our model represents an example of a situation in which physically interesting noncommutative particle emerges in this way. In our formalism, components of the position variable have non-trivial Dirac brackets originating from the necessity to take into account the spin-tensor condition $J^{\mu \nu} \dot{x}_{\mu}=0$. In the result, we deal with a noncommutative position coordinate instead of the canonical one. The description of spin on the base of vector-like variables leads inevitably to the position noncommutativity, eq. (3.22).

In general relativity, a consistent definition of quantum spin is a nontrivial task [52]. We note that with the variational formulation at hand, quantization of a theory represents a more or less straightforward procedure, thus opening the way to further studies of microscopic phenomena involving spin, gravity and inertia [53]. For instance, accounting for spin-rotation coupling [54] is important for precise measurements of anomalous magnetic momentum of elementary particles [55].

\section{A Dirac brackets}

We construct Dirac brackets that take into account the subset of constraints $\left\{\Psi_{a}\right\}$ with non degenerated second-class constraint matrix (3.20). The Dirac bracket between any two 
phase space functions $A$ and $B$ is given by (3.21). For the basic variables we obtain

$$
\begin{aligned}
\left\{x^{\mu}, x^{\nu}\right\}_{D}= & \frac{J^{\mu \nu}}{2 M^{2}}, \\
\left\{x^{\mu}, P_{\nu}\right\}_{D}= & \delta_{\nu}^{\mu}-\frac{1}{8 M}\left[\theta_{\alpha \nu} J^{\mu \alpha}+4 \Gamma_{\nu \beta}^{\alpha} P_{\alpha} J^{\beta \mu}\right] \\
\left\{x^{\mu}, \omega^{\nu}\right\}_{D}= & \frac{\omega^{\mu} P^{\nu}}{M^{2}}+\frac{1}{2 M^{2}} \Gamma_{\alpha \beta}^{\nu} \omega^{\beta} J^{\alpha \mu} \\
\left\{x^{\mu}, \pi^{\nu}\right\}_{D}= & \frac{\pi^{\mu} P^{\nu}}{M^{2}}-\frac{1}{2 M^{2}} \Gamma_{\alpha \beta}^{\nu} \pi^{\alpha} J^{\mu \beta} \\
\left\{x^{\mu}, J^{\alpha \beta}\right\}_{D}= & \frac{P^{[\beta} J^{\alpha] \mu}}{M^{2}}+\frac{1}{2 M^{2}} J^{\mu \sigma} \Gamma_{\sigma \nu}^{[\alpha} J^{\beta] \nu} \\
\left\{P_{\mu}, P_{\nu}\right\}_{D}= & \frac{1}{4} \theta_{\mu \nu}-\frac{\theta_{\alpha \mu} \theta_{\beta \nu} J^{\beta \alpha}}{32 M^{2}} \\
& +\frac{1}{4 M^{2}}\left[\theta_{\alpha \mu} \Gamma_{\nu \beta}^{\lambda} P_{\lambda} J^{\alpha \beta}+\theta_{\beta \nu} \Gamma_{\mu \alpha}^{\lambda} P_{\lambda} J^{\alpha \beta}+4 \Gamma_{\mu \beta}^{\alpha} \Gamma_{\nu \sigma}^{\lambda} P_{\alpha} P_{\lambda} J^{\sigma \beta}\right] \\
\left\{P_{\mu}, \omega^{\nu}\right\}_{D}= & \Gamma_{\mu \alpha}^{\nu} \omega^{\alpha}-\frac{1}{4}\left[\theta_{\alpha \mu} \omega^{\alpha} P^{\nu}+\frac{1}{2} \theta_{\alpha \mu} \Gamma_{\sigma \lambda}^{\nu} \omega^{\lambda} J^{\sigma \alpha}-4 \Gamma_{\mu \beta}^{\alpha} \Gamma_{\sigma \lambda}^{\nu} P_{\alpha} \pi^{\beta} \omega^{\sigma} \omega^{\lambda}\right] \\
\left\{P_{\mu}, \pi_{\nu}\right\}_{D}= & -\Gamma_{\mu \nu}^{\alpha} \pi_{\alpha}-\frac{1}{4}\left[\theta_{\alpha \mu} \pi^{\alpha} P_{\nu}+\frac{1}{2} \theta_{\alpha \mu} \Gamma_{\nu \lambda}^{\sigma} \pi_{\sigma} J^{\alpha \lambda}-4 \Gamma_{\mu \beta}^{\alpha} \Gamma_{\nu \lambda}^{\sigma} P_{\alpha} \omega^{\beta} \pi^{\lambda} \pi_{\sigma}\right] \\
\left\{\omega^{\mu}, \pi_{\nu}\right\}_{D}= & \delta_{\nu}^{\mu}-\frac{\pi^{\mu} \pi_{\nu}}{a_{3}} \\
& +\frac{1}{M^{2}}\left[\Gamma_{\alpha \beta}^{\mu} \omega^{\alpha} \omega^{\beta} \Gamma_{\nu \lambda}^{\sigma} \pi_{\sigma} \pi^{\lambda}+\left(P^{\mu}-\Gamma_{\alpha \beta}^{\mu} \pi^{\alpha} \omega^{\beta}\right)\left(P_{\nu}-\Gamma_{\nu \lambda}^{\sigma} \omega^{\lambda} \pi_{\sigma}\right]\right.
\end{aligned}
$$

The tensor $\theta_{\mu \nu}$ is defined in (4.8).

\section{Acknowledgments}

The work of AAD has been supported by the Brazilian foundation CNPq. AMPM and WGR thanks CAPES for the financial support (Programm PNPD/2011).

Open Access. This article is distributed under the terms of the Creative Commons Attribution License (CC-BY 4.0), which permits any use, distribution and reproduction in any medium, provided the original author(s) and source are credited.

\section{References}

[1] V.A. Fock, Sur le mouvement des masses finies d'apres la théorie de gravitation einsteinienne, J. Phys. Acad. Sci. USSR 1 (1939) 81.

[2] A. Papapetrou, Spinning test particles in general relativity. 1, Proc. Roy. Soc. Lond. A 209 (1951) 248 [inSPIRE].

[3] S. Goudschmidt and G. Uhlenbeck, Spinning electrons and the structure of spectra, Nature 117 (1926) 264 [INSPIRE].

[4] J. Frenkel, Die Elektrodynamik des rotierenden Elektrons, Z. Phys. 37 (1926) 243 [InSPIRE].

[5] V. Bargmann, L. Michel and V. Telegdi, Precession of the polarization of particles moving in a homogeneous electromagnetic field, Phys. Rev. Lett. 2 (1959) 435 [INSPIRE]. 
[6] H.C. Corben, Classical and quantum theories of spinning particles, Holden-Day, San Francisco U.S.A. (1968).

[7] A.J. Hanson and T. Regge, The relativistic spherical top, Ann. Phys. 87 (1974) 498 [INSPIRE].

[8] P. Grassberger, Classical charged particles with spin, J. Phys. A 11 (1978) 1221 [InSPIRE].

[9] G. Cognola, L. Vanzo, S. Zerbini and R. Soldati, On the lagrangian formulation of a charged spinning particle in an external electromagnetic field, Phys. Lett. B 104 (1981) 67 [INSPIRE].

[10] F.A. Berezin and M.S. Mariñov, Particle spin dynamics as the Grassmann variant of classical mechanics, Annals Phys. 104 (1977) 336 [INSPIRE].

[11] R.H. Rietdijk and J.W. van Holten, Spinning particles in Schwarzschild space-time, Class. Quant. Grav. 10 (1993) 575 [INSPIRE].

[12] F. Ravndal, Supersymmetric Dirac particles in external fields, Phys. Rev. D 21 (1980) 2823 [INSPIRE].

[13] A.A. Deriglazov, Spinning-particle model for the Dirac equation and the relativistic Zitterbewegung, Phys. Lett. A 376 (2012) 309 [arXiv:1106.5228] [INSPIRE].

[14] A.A. Deriglazov, Variational problem for the Frenkel and the Bargmann-Michel-Telegdi (BMT) equations, Mod. Phys. Lett. A 28 (2013) 1250234 [arXiv:1204.2494] [INSPIRE].

[15] A.A. Deriglazov and A.M. Pupasov-Maksimov, Frenkel electron on an arbitrary electromagnetic background and magnetic Zitterbewegung, arXiv:1401.7641 [INSPIRE].

[16] A. Deriglazov and A. Nersessian, Rigid particle revisited: extrinsic curvature yields the Dirac equation, arXiv:1303.0483 [INSPIRE].

[17] A.A. Deriglazov and A.M. Pupasov-Maksimov, Lagrangian for Frenkel electron and position's non-commutativity due to spin, arXiv:1312.6247 [INSPIRE].

[18] M. Mathisson, Neue mechanik materieller systemes, Acta Phys. Polon. 6 (1937) 163 [INSPIRE].

[19] A.A. Pomeranskiǔ, R. A, Sen'kov and I.B. Khriplovich, Spinning relativistic particles in external fields, Phys. Usp. 43 (2000) 1055.

[20] E. Corinaldesi and A. Papapetrou, Spinning test particles in general relativity. 2, Proc. Roy. Soc. Lond. A 209 (1951) 259 [inSPIRE].

[21] O. Semerak, Spinning test particles in a Kerr field. 1, Mon. Not. Roy. Astron. Soc. 308 (1999) 863 [INSPIRE].

[22] R. Plyatsko and M. Fenyk, Highly relativistic circular orbits of spinning particle in the Kerr field, Phys. Rev. D 87 (2013) 044019 [arXiv: 1303.4707] [INSPIRE].

[23] M. Mohseni, Spinning particles in gravitational wave space-time, Phys. Lett. A 301 (2002) 382 [gr-qc/0208072] [InSPIRE].

[24] T.W. Kornack, R.K. Ghosh and M.V. Romalis, Nuclear spin gyroscope based on an atomic comagnetometer, Phys. Rev. Lett. 95 (2005) 230801 [physics/0505089].

[25] C. Chicone, B. Mashhoon and B. Punsly, Relativistic motion of spinning particles in a gravitational field, Phys. Lett. A 343 (2005) 1 [gr-qc/0504146] [INSPIRE].

[26] S. Hojman, Lagrangian theory of the motion of spinning particles in torsion gravitational theories, Phys. Rev. 18 (1978) 2741. 
[27] R.A. Porto, Post-Newtonian corrections to the motion of spinning bodies in NRGR, Phys. Rev. D 73 (2006) 104031 [gr-qc/0511061] [INSPIRE].

[28] K. Yee and M. Bander, Equations of motion for spinning particles in external electromagnetic and gravitational fields, Phys. Rev. D 48 (1993) 2797 [hep-th/9302117] [INSPIRE].

[29] G. Burdet, M. Perrin and M. Perroud, Universal description of spinning test particles, Gen. Rel. Grav. 24 (1992) 773.

[30] A.A. Deriglazov and A.M. Pupasov-Maksimov, Geometric constructions underlying relativistic description of spin on the base of non-Grassmann vector-like variable, SIGMA 10 (2014) 012 [arXiv:1311.7005] [INSPIRE].

[31] A. Deriglazov, Variational problem for Hamiltonian system on so $(k, m)$ Lie-Poisson manifold and dynamics of semiclassical spin, arXiv:1211.1219 [INSPIRE].

[32] J.W. van Holten, On the electrodynamics of spinning particles, Nucl. Phys. B 356 (1991) 3.

[33] P.L. Nash, A lagrangian theory of the classical spinning electron, J. Math. Phys. 25 (1984) 2104.

[34] D.M. Gitman and I.V. Tyutin, Quantization of fields with constraints, Springer-Verlag, Berlin Germany (1990).

[35] A. Deriglazov, Poincaré covariant mechanics on noncommutative space, JHEP 03 (2003) 021 [hep-th/0211105] [INSPIRE].

[36] A.A. Deriglazov, Noncommutative relativistic particle on the electromagnetic background, Phys. Lett. B 555 (2003) 83 [hep-th/0208201] [INSPIRE].

[37] E.M.C. Abreu, R. Amorim and W.G. Ramirez, Noncommutative particles in curved spaces, JHEP 03 (2011) 135 [arXiv: 1011.0023] [INSPIRE].

[38] R. Amorim, E.M.C. Abreu and W.G. Ramirez, Noncommutative relativistic particles, Phys. Rev. D 81 (2010) 105005 [arXiv:1001.2178] [InSPIRE].

[39] M. Daszkiewicz, Equivalent forces in Newton equation from twist deformations and noninertial coordinate frames, Mod. Phys. Lett. A 28 (2013) 1350154 [arXiv:1310.5427] [INSPIRE].

[40] M. Daszkiewicz, Quantum mechanics of many particles defined on twisted $N$-enlarged Newton-Hooke space-times, Acta Phys. Polon. B 44 (2013) 699 [arXiv:1304.6801] [INSPIRE].

[41] J. Alfaro and V.O. Rivelles, Very special relativity and Lorentz violating theories, arXiv:1306.1941 [INSPIRE].

[42] S. Deguchi, S. Negishi, S. Okano and T. Suzuki, Canonical formalism and quantization of a massless spinning bosonic particle in four dimensions, arXiv:1309.4169 [INSPIRE].

[43] M. Eune and W. Kim, Remarks on generalized uncertainty principle induced from constraint system, Mod. Phys. Lett. A 29 (2014) 1450002 [arXiv:1309.0588] [InSPIRE].

[44] P.A. Horvathy, Mathisson' spinning electron: noncommutative mechanics and exotic Galilean symmetry, 66 years ago, Acta Phys. Polon. B 34 (2003) 2611 [hep-th/0303099] [InSPIRE].

[45] A. Deriglazov, Classical mechanics: hamiltonian and lagrangian formalism, Springer-Verlag, Germany (2010). 
[46] I. Khriplovich and A. Pomeransky, Gravitational interaction of spinning bodies, center-of-mass coordinate and radiation of compact binary systems, Phys. Lett. A 216 (1996) 7 [gr-qc/9602004] [INSPIRE].

[47] B.M. Barker and R.F. O'Connell, Nongeodesic motion in general relativity, Gen. Rel. Grav. 5 (1974) 539.

[48] B.M. Barker and R.F. O'Connell, Derivation of the equations of motion of a gyroscope from the quantum theory of gravitation, Phys. Rev. D 2 (1970) 1428.

[49] B.M. Barker and R.F. O'Connell, The gravitational interaction: spin, rotation, and quantum effects, Gen. Rel. Grav. 11 (1979) 149.

[50] A.A. Deriglazov, Classical-mechanical models without observable trajectories and the Dirac electron, Phys. Lett. A 377 (2012) 13 [arXiv:1203.5697] [InSPIRE].

[51] A.A. Deriglazov, Semiclassical description of relativistic spin without use of Grassmann variables and the Dirac equation, Annals Phys. 327 (2012) 398 [arXiv:1107.0273] [INSPIRE].

[52] D. Singh and N. Mobed, The implications of noninertial motion on covariant quantum spin, Class. Quant. Grav. 24 (2007) 2453 [hep-th/0506156] [INSPIRE].

[53] B. Mashhoon, Gravitational couplings of intrinsic spin, Class. Quant. Grav. 17 (2000) 2399 [gr-qc/0003022] [INSPIRE].

[54] G. Lambiase and G. Papini, Spin-rotation coupling in compound spin objects, Phys. Lett. A 377 (2013) 1021 [arXiv:1301.7561] [InSPIRE].

[55] G. Papini and G. Lambiase, Spin rotation coupling in muon $g-2$ experiments, Phys. Lett. A 294 (2002) 175 [gr-qc/0106066] [INSPIRE]. 